\title{
Complicaciones intraoperatorias en cirugía endoscópica nasosinusal en Servicio de Otorrinolaringología del Hospital del Salvador, revisión en los últimos 10 años
}

\author{
Intraoperative complications in endoscopic sinus surgery in \\ otorhinolaryngology service at Hospital del Salvador, review \\ in the last 10 years
}

\author{
Paula Silva R. ${ }^{1}$, Daniela Vicencio S. ${ }^{1}$, Tamara Barría E. ${ }^{2,3}$, Paula Ruz M..$^{2,4}$, Marcela Veloz T. ${ }^{2,5}$, \\ Carlos Tapia M..$^{2,3}$, Samanta Ruz G., ${ }^{2,6}$ Constanza Valdés $P^{2,4}$
}

${ }^{1}$ Servicio de

Otorrinolaringología, Hospital Carlos Van Buren. Viña del

Mar, Chile.

${ }^{2}$ Servicio de

Otorrinolaringología, Hospita del Salvador. Santiago, Chile.

${ }^{3}$ Servicio de

Otorrinolaringología, Clínica

Dávila. Santiago, Chile.

${ }^{4}$ Servicio de

Otorrinolaringología, Clínica Las Condes. Santiago, Chile.

${ }^{5}$ Servicio de

Otorrinolaringología, Clínica Santa María. Santiago, Chile.

${ }^{6}$ Servicio de

Otorrinolaringología, Clínica Indisa. Santiago, Chile.

Los autores declaran no tener conflictos de interés.

Recibido el 2 de agosto de 2020. Aceptado el 25 de abril de 2021

Correspondencia: Constanza Valdés $P$. Avenida Salvador \#364, Providencia. Santiago, Chile. Email: cvaldesp@clc.c

\section{Resumen}

Introducción: La cirugía endoscópica nasal (CEN) corresponde a una técnica quirúrgica mínimamente invasiva. Ha disminuido la morbimortalidad secundaria al acto quirúrgico. Pese a esto, no está exenta de riesgos y sus complicaciones pueden variar en severidad desde leves hasta catastróficas, siendo la hemorragia nasal la más frecuente. Objetivo: Analizar las complicaciones intraoperatorias, factores asociados a complicaciones y manejo en nuestra realidad local. Material y Método: Estudio retrospectivo, revisión de protocolos operatorios de pacientes atendidos en el Hospital del Salvador entre los años 2009 y 2019. Resultados: Se revisaron 602 cirugías de las cuales se excluyeron 18. De un total de 584 CEN realizadas durante los últimos diez años, la incidencia de complicaciones intraoperatorias fue de 3,3\%. Sólo se observaron complicaciones hemorrágicas $(2 \%)$ y orbitarias $(1,2 \%)$. La incidencia de complicaciones mayores fue $0,51 \%$. La única variable asociada de forma significativa con la presencia de complicación intraoperatoria fue el tiempo quirúrgico. Conclusión: Como centro presentamos una tasa de complicaciones intraoperatorias de CEN baja en los últimos diez años, dentro de las cuales las más frecuentes son las hemorrágicas y las orbitarias; comparables con la literatura internacional. Se establece el primer reporte a nivel nacional sobre las complicaciones intraoperatorias de CEN.

Palabras clave: complicaciones, cirugía endoscópica nasal, hemorragia, rinosinusitis.

\begin{abstract}
Introduction: Endoscopic sinus surgery (ESS) is a minimally invasive surgical technique. It has decreased morbidity and mortality secondary to the surgical act. Despite this, endoscopic sinus surgery is not a risk-free procedure and its complications can range from mild to severe, with nosebleed being the most frequent. Aim: To analyze the surgical results of nasal endoscopic surgery, with its intraoperative complications and management in our local reality. Material and Method: Retrospective study, review of operative protocols of patients attended at the Salvador Hospital between 2009 and 2019. Results: 602 surgeries were reviewed, of which 18 were excluded. Of a total of 584 ESS performed during the last ten years, the incidence of intraoperative complications was 3,3\%. Only hemorrhagic (2\%) and orbital complications (1,2\%) were observed. The incidence of major complications was $0,51 \%$. The only variable significantly associated with the presence of intraoperative complications was surgical time. Conclusion: As a center, we present a low rate of intraoperative complications of ESS in the last ten years, among which the most frequent are hemorrhagic and orbital; comparable to international literature. The first national report on intraoperative complications of ESS is established.
\end{abstract}

Keywords: complications, endoscopic sinus surgery, bleeding, rhinosinusitis 


\section{Introducción}

La cirugía endoscópica nasal es una técnica quirúrgica mínimamente invasiva que tiene como objetivo facilitar la ventilación, restaurar el drenaje mucociliar y facilitar la distribución adecuada del tratamiento tópico en las cavidades paranasales ${ }^{1}$. En la actualidad las indicaciones de esta técnica abarcan distintos procesos no inflamatorios de nariz y senos paranasales como: patología tumoral, atresia de coanas, lesiones de la base de cráneo, descompresión orbitaria, entre otros; todo esto de una forma más individualizada y menos invasiva, evitando así procedimientos abiertos y radicales ${ }^{2}$. Como todo procedimiento quirúrgico no se encuentra exento de complicaciones, las que se clasifican en mayores y menores. Esta división fue planteada inicialmente por Stankiewicz ${ }^{3-5} y$ ha sido adaptada con el transcurso de los años como se observa en la Tabla 1.

Complicaciones menores y mayores de cirugía endoscópica nasal. Adaptación de Vargas-Aguayo y cols. $(2014)^{6}$. Cirugía endoscópica de nariz y senos paranasales: Complicaciones en 150 pacientes. Revista Médica del Instituto Mexicano del Seguro Social.

$\mathrm{La}$ incidencia global de complicaciones asociadas a CEN se describe cercana al 5\%, la que es mucho menor y cercana al 1\% cuando se trata de complicaciones mayores ${ }^{7}$. La incidencia de cada una de estas complicaciones en particular varía según el estudio analizado de acuerdo a su metodología y seguimiento, como se puede apreciar en la Tabla 2, en la que se observa que las complicaciones mayores fluctúan entre 0,4\%-8\% y las menores entre 6,9\%-37\%.

Se han descrito factores asociados a mayor riesgo de complicación, partiendo por el grado de extensión de la lesión o patología ${ }^{10}$, su lateralidad (mayor riesgo a derecha) ${ }^{12}$ y si se trata esta de una reintervención ${ }^{3}$. Existen factores intrínsecos del paciente como su anatomía y presencia de comorbilidades ${ }^{10-12}$. Con respecto a los factores del cirujano hay una clara la relación con la curva de aprendizaje, es importante destacar que las complicaciones tienen a disminuir a lo largo de los años, pero no desaparecen ${ }^{1,3}$. Por último, existe relación directa con el tipo de anestesia utilizada, debido al efecto vasodilatador de esta cuando no se cuenta con anestesia total intravenosa $(\text { TIVA })^{3,13}$. A nivel chileno, no existen reportes sobre este tipo de complicaciones a la fecha de esta publicación.

\section{Objetivo}

El objetivo de este trabajo es analizar las complicaciones intraoperatorias, factores asociados a complicaciones y manejo en nuestra realidad local en CEN en el Hospital del Salvador de Santiago de Chile en el periodo 2009-2019.

Tabla 1. Clasificación de complicaciones en CEN

\begin{tabular}{ll}
\hline Complicaciones menores & Complicaciones mayores \\
\hline Hemorragia $(<500 \mathrm{~mL})$ & Hemorragia $(>500 \mathrm{ml})$ \\
\hline Hiposmia & Trauma del conducto nasolagrimal \\
\hline Sinequia & Lesión carotídea \\
\hline Cefalea & Hemorragia intracraneal \\
\hline Equimosis & Hematoma orbitario \\
\hline Enfisema periorbitario & Diplopía \\
\hline Dolor/adormecimiento facial o dental & Disminución de la agudeza visual \\
\hline & Ceguera \\
\hline & Fístula de líquido cerebroespinal \\
\hline Neumoencéfalo \\
\hline
\end{tabular}


Tabla 2. Reportes de complicaciones en CEN

\begin{tabular}{|c|c|c|c|}
\hline Estudio & Pacientes & Complicaciones mayores & Complicaciones menores \\
\hline $\begin{array}{l}\text { Stankiewicz }{ }^{3} \text {. Complications } \\
\text { of endoscopic intranasal } \\
\text { ethmoidectomy, } 1987\end{array}$ & 90 casos & $\begin{array}{l}\text { Total: } 8 \% \\
\text { - Hemorragia: 1,8\% } \\
\text { - Fístula LCE: 0,9\% } \\
\text { - Ceguera: 0,9\% }\end{array}$ & $\begin{array}{l}\text { Total: } 21 \% \\
\text { - Enfisema orbitario: } 2,7 \% \\
\text { - Sinequias: 5,4\% } \\
\text { - Cierre de meatotomía: 3,6\% } \\
\text { - Dolor dental: } 0,9 \%\end{array}$ \\
\hline $\begin{array}{l}\text { May y cols. }{ }^{8} \\
\text { Complications of Endoscopic } \\
\text { sinus surgery: Analysis of } \\
2108 \text { patients-Incidence and } \\
\text { Prevention, } 1994\end{array}$ & 2.108 casos & $\begin{array}{l}\text { Total: } 0,85 \% \\
\text { - Hemorragia: 0,19\% } \\
\text { - Orbitarias: 0,05\% } \\
\text { - Lacrimales: } 0,14 \% \\
\text { - intracraneales: } 0,47 \%\end{array}$ & $\begin{array}{l}\text { Total: } 6,9 \% \\
\text { - Complicaciones orbitarias: } \\
1,7 \% \\
\text { - Epistaxis: } 0,6 \% \\
\text { - Broncoespasmo: } 1,6 \% \\
\text { - Sinequias: } 1,7 \% \\
\text { - Otras no especificadas: } 6,9 \%\end{array}$ \\
\hline $\begin{array}{l}\text { Dessi y cols. }{ }^{9} \\
\text { Major complications of sinus } \\
\text { surgery: a review of } 1192 \\
\text { procedures, } 1994\end{array}$ & 1.192 casos & $\begin{array}{l}\text { Total: } 0,4 \% \\
\text { - Hematoma órbito palpebral: } \\
0,3 \% \\
\text { - Fístula LCR: } 0,15 \%\end{array}$ & No descrito \\
\hline $\begin{array}{l}\text { Bross y cols. }^{2} \\
\text { Complicaciones y causas de } \\
\text { falla en cirugía endoscópica } \\
\text { nasosinusal, } 1999\end{array}$ & 220 casos & $\begin{array}{l}\text { Total: } 0,45 \% \\
\text { - Hemorragia: 0,45\% }\end{array}$ & $\begin{array}{l}\text { Total: } 9,09 \% \\
\text { - Hematomas subpalpebrales: } \\
1,82 \% \\
\text { - Sinequias: } 7,27 \%\end{array}$ \\
\hline $\begin{array}{l}\text { Lobo y cols. }^{1} \\
\text { Cirugía endoscópica } \\
\text { nasosinusal. Revisión de } 1.093 \\
\text { casos, } 2003\end{array}$ & 1.093 casos & $\begin{array}{l}\text { Total: } 0,82 \% \\
\text { - Fístula LCE: 0,82\% }\end{array}$ & $\begin{array}{l}\text { Total: } 37,8 \% \\
\text { - Equimosis palpebral: 3,75\% } \\
\text { - Hemorragia intraoperatoria: } \\
\text { 7,95\% } \\
\text { - Epistaxis posoperatoria: 9,8\% } \\
\text { - Crisis asmática posoperatoria: } \\
\text { 1,92\% } \\
\text { - Sinequias: } 7 \% \\
\text { - Cierre de meatotomía: } 8,14 \%\end{array}$ \\
\hline $\begin{array}{l}\text { Hopkins y cols. }{ }^{10} \\
\text { Complications of Surgery for } \\
\text { Nasal Polyposis and Chronic } \\
\text { Rhinosinusitis: The results of a } \\
\text { national audit in England and } \\
\text { Wales, } 2006\end{array}$ & 3.128 casos & $\begin{array}{l}\text { Total: } 0,4 \% \\
\text { - Orbitarias: 0,2\% } \\
\text { - Intracraneales: } 0,06 \% \\
\text { - Hemorragia masiva: } 0,06 \%\end{array}$ & $\begin{array}{l}\text { Total: } 6,6 \% \\
\text { - Hemorragia: } 0,8 \% \\
\text { - Lesión de lámina papirácea: } \\
\text { 0,4\% }\end{array}$ \\
\hline $\begin{array}{l}\text { Guerrero y cols. }{ }^{11} \\
\text { Cirugía endoscópica } \\
\text { nasosinusal: rinosinusitis } \\
\text { crónica con pólipos, } 2007\end{array}$ & 110 casos & No hubo & $\begin{array}{l}\text { Total: } 19 \% \\
\text { - Equimosis: } 6,4 \% \\
\text { - Hemorragia: } 4,5 \% \\
\text { - Sinequias: } 9,1 \%\end{array}$ \\
\hline
\end{tabular}

LCE: líquido cerebroespinal.

\section{Material y Método}

Estudio retrospectivo, se realizó una revisión de protocolos operatorios de pacientes operados de cirugía endoscópica nasal en el Hospital del Salvador entre los años 20092019. Dentro de los criterios de exclusión se encuentran aquellas cirugías que no presen- taron apertura de alguna cavidad paranasal, como toma de biopsia, septoplastia y/o turbinoplastía por sí solas.

Con respecto a las complicaciones hemorrágicas, se incluyeron aquellas que fueran de una cuantía de $>500 \mathrm{ml}$, que tuvieran repercusión hemodinámica y/o fueran causante de término una cirugía antes de lo previsto. 
Se formó un registro de datos en relación con el diagnóstico, presencia de complicación, lateralidad de ésta y al tiempo quirúrgico reportados en protocolos quirúrgicos. Se hizo un análisis exploratorio de datos, calculando valores absolutos y medidas de tendencia central, para las respectivas variables. Se estimó la presencia de asociaciones entre la presencia de complicaciones quirúrgicas, el diagnóstico y la lateralidad de la complicación utilizando el test exacto de Fisher, y la asociación entre la ocurrencia de una complicación con el tiempo quirúrgico, mediante el test de Mann-Whitney. Esta revisión fue aprobada por el comité de ética del Servicio de Salud Metropolitano Oriente según la normativa vigente.

\section{Resultados}

Se obtuvo un total de 602 cirugías, de las cuales se excluyeron 18: 11 por falta de datos y 7 en los que solo se tomó biopsia. En la Figura 1 se observan los diagnósticos asociados a las 584 cirugías analizadas. El 62\% correspondió a rinosinusitis crónica (RSC), de ellas, el 69\% se trató de RSC primaria difusa con pólipos; les siguen los tumores nasales (19\%) y mucoceles (5\%). Con menor frecuencia se encuentran: RSC localizada secundaria a bola fúngica $(2,4 \%)$ rinosinusitis fúngica invasiva aguda $(3,8 \%)$, epistaxis $(0,3 \%)$ y otros diagnósticos $(3,9 \%)$.
Durante los diez años de observación se reportaron 20 complicaciones intraoperatorias, de las cuales 19 fueron quirúrgicas (3,25\%) y 1 médica $(0,17 \%)$, que correspondió a un shock anafiláctico manejado de forma exitosa por el equipo de anestesia. Con respecto a las complicaciones quirúrgicas se reportaron 12 cirugías con complicaciones del tipo orbitarias y 7 cirugías con complicaciones hemorrágicas.

\section{a) Complicaciones orbitarias}

Se reportaron 12 casos (Tabla 3), lo que corresponde a una incidencia global de 2,05\%. Existe un caso de hematoma orbitario en un paciente con tumor nasal maligno, siendo esta la única complicación orbitaria mayor de nuestra serie, representando una incidencia de $0,17 \%$. Los otros 11 casos presentaron dehiscencia de lámina papirácea con exposición de grasa periorbitaria hacia la cavidad nasal, secundaria al acto quirúrgico. Las patologías asociadas a complicaciones orbitarias fueron RSC primaria difusa (no poliposa 4 casos, poliposa 3 casos y complicada 1 caso) y los tumores nasales (3 casos). Ningún paciente evolucionó con secuelas visuales.

\section{b) Complicaciones hemorrágicas}

La incidencia fue de 1,2\% con 7 pacientes que se presentan en la Tabla 4, de los cuales, 2 casos requirieron transfusión intraoperatoria y 3 fueron causantes de cirugía incompleta. La principal patología que se asoció a complica-

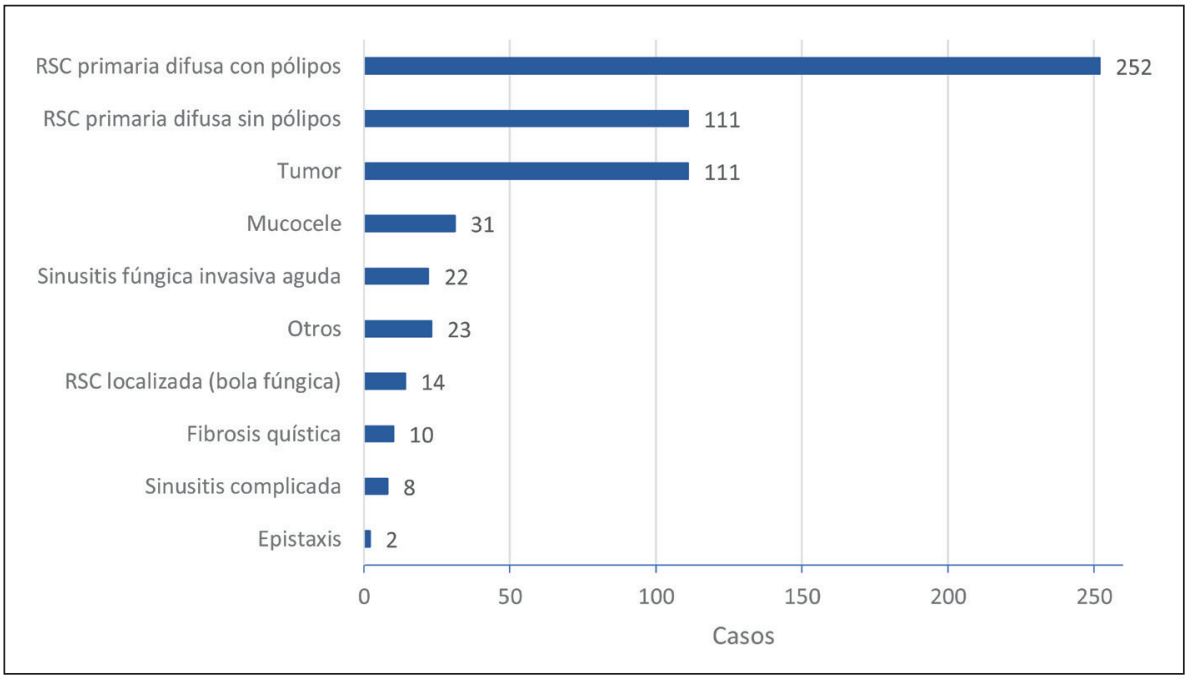

Figura 1. Diagnósticos de las cirugías analizadas (número de casos). 


\begin{tabular}{|c|c|c|c|c|c|c|}
\hline Paciente & Patología & $\begin{array}{l}\text { CEN de } \\
\text { revisión }\end{array}$ & Complicación & $\begin{array}{c}\text { Tiempo } \\
\text { quirúrgico }(\mathrm{min})\end{array}$ & $\begin{array}{l}\text { Lateralidad } \\
\text { de cirugía }\end{array}$ & Tratamiento \\
\hline Caso 8 & $\begin{array}{l}\text { RSC PD sin } \\
\text { pólipos }\end{array}$ & No & $\begin{array}{l}\text { Exposición grasa } \\
\text { periorbitaria }\end{array}$ & 150 & Bilateral & No \\
\hline Caso 9 & $\begin{array}{l}\text { RSC PD con } \\
\text { pólipos }\end{array}$ & No & $\begin{array}{l}\text { Exposición grasa } \\
\text { periorbitaria }\end{array}$ & 150 & Bilateral & No \\
\hline Caso 10 & $\begin{array}{l}\text { RSC PD con } \\
\text { pólipos }\end{array}$ & No & $\begin{array}{c}\text { Exposición grasa } \\
\text { periorbitaria }\end{array}$ & 240 & Bilateral & No \\
\hline Caso 11 & $\begin{array}{l}\text { Papiloma } \\
\text { invertido }\end{array}$ & No & $\begin{array}{c}\text { Exposición grasa } \\
\text { periorbitaria }\end{array}$ & 180 & Bilateral & No \\
\hline Caso 12 & $\begin{array}{l}\text { RSC PD con } \\
\text { pólipos }\end{array}$ & No & $\begin{array}{c}\text { Exposición grasa } \\
\text { periorbitaria }\end{array}$ & 139 & Bilateral & No \\
\hline Caso 13 & $\begin{array}{l}\text { RSC PD con } \\
\text { pólipos }\end{array}$ & No & $\begin{array}{c}\text { Exposición grasa } \\
\text { periorbitaria }\end{array}$ & 145 & Bilateral & No \\
\hline Caso 14 & $\begin{array}{l}\text { RSC PD sin } \\
\text { pólipos }\end{array}$ & No & $\begin{array}{c}\text { Exposición grasa } \\
\text { periorbitaria }\end{array}$ & 230 & Bilateral & No \\
\hline Caso 15 & $\begin{array}{l}\text { RSC PD sin } \\
\text { pólipos }\end{array}$ & No & $\begin{array}{c}\text { Exposición grasa } \\
\text { periorbitaria }\end{array}$ & 195 & Bilateral & No \\
\hline Caso 16 & $\begin{array}{l}\text { RSC PD sin } \\
\text { pólipos }\end{array}$ & No & $\begin{array}{c}\text { Exposición grasa } \\
\text { periorbitaria }\end{array}$ & 145 & Bilateral & No \\
\hline Caso 17 & $\begin{array}{l}\text { Papiloma } \\
\text { Invertido }\end{array}$ & No & $\begin{array}{l}\text { Exposición grasa } \\
\text { periorbitaria }\end{array}$ & 240 & Bilateral & No \\
\hline Caso 18 & $\begin{array}{l}\text { RSC PD con } \\
\text { pólipos }\end{array}$ & No & $\begin{array}{l}\text { Exposición grasa } \\
\text { periorbitaria }\end{array}$ & 180 & Bilateral & No \\
\hline Caso 19 & $\begin{array}{c}\text { Tumor } \\
\text { maligno nasal }\end{array}$ & No & $\begin{array}{c}\text { Hematoma } \\
\text { orbitario derecho }\end{array}$ & 150 & Unilateral & $\begin{array}{c}\text { Cauterio, retiro de } \\
\text { tapones, cantotomía } \\
\text { y cantolisis }\end{array}$ \\
\hline Caso 20 & $\begin{array}{l}\text { RSC localizada } \\
\text { (bola fúngica) }\end{array}$ & No & $\begin{array}{c}\text { Exposición grasa } \\
\text { periorbitaria }\end{array}$ & 80 & Unilateral & No \\
\hline
\end{tabular}

CEN: cirugía endoscópica nasal; RSC PD: rinosinusitis crónica primaria difusa; RSC rinosinusitis crónica.

ción hemorrágica fue la RSC primaria difusa con pólipos, además de un caso de rinosinusitis fúngica invasiva aguda y un caso de nasofibroangioma, este último a pesar de haber sido embolizado $48 \mathrm{~h}$ previo a la cirugía.

\section{Factores asociados a las complicaciones}

Al realizar el análisis estadístico de las variables asociadas, la única que se asoció de forma significativa a mayor frecuencia de complicación intraoperatoria fue el tiempo quirúrgico $(\mathrm{p}<0,05)$, siendo mayor en las cirugías con complicación. El promedio de las cirugías sin complicación fue de 150 minutos (rango 30-425 min; DS 59,7), mientras que las cirugías con complicación presentaron un tiempo promedio de 180 minutos, lo que es significativo según test de Fisher. Por el contrario, al analizar la lateralidad de las complicaciones o las patologías asociadas a estas, no se obtuvo asociación estadísticamente significativa $(p>0,05)$.

\section{Discusión}

En base al análisis estadístico de las cifras del Hospital del Salvador, se observa una incidencia total de complicaciones intraoperatorias de 3,3\%, lo cual es menor a la litera- 


\begin{tabular}{|c|c|c|c|c|c|}
\hline Pacientes & Patología & $\begin{array}{l}\text { CEN de } \\
\text { revisión }\end{array}$ & $\begin{array}{c}\text { Tiempo } \\
\text { quirúrgico (min) }\end{array}$ & $\begin{array}{l}\text { Lateralidad de } \\
\quad \text { cirugía }\end{array}$ & Tratamiento \\
\hline Caso 1 & RSC PD con pólipos & No & 120 & Unilateral & $\begin{array}{c}\text { Merocel } \\
\text { Cirugía incompleta }\end{array}$ \\
\hline Caso 2 & Nasofibroangioma & No & 210 & $\begin{array}{c}\text { Unilateral con } \\
\text { ventana transeptal }\end{array}$ & $\begin{array}{l}\text { Surgiflo y surgicel + } \\
\text { transfusión GR }\end{array}$ \\
\hline Caso 3 & RS fúngica invasiva & No & 165 & Bilateral & $\begin{array}{c}\text { Gasa y merocel } \\
\text { Cirugía incompleta }\end{array}$ \\
\hline Caso 4 & RSC PD con pólipos & No & 360 & Bilateral & Cauterio, transfusión GR \\
\hline Caso 5 & RSC PD con pólipos & No & 225 & Bilateral & Cauterio \\
\hline Caso 6 & RSC PD con pólipos & No & 200 & Bilateral & $\begin{array}{c}\text { Cauterio, merocel y cirugía } \\
\text { incompleta }\end{array}$ \\
\hline Caso 7 & RSC PD con pólipos & No & 85 & Bilateral & $\begin{array}{c}\text { Gasa con vasoconstrictores, } \\
\text { cirugía incompleta }\end{array}$ \\
\hline
\end{tabular}

tura internacional en las que se manejan tasas cercanas al $5 \%{ }^{7}$.

Con respecto a las complicaciones mayores según la clasificación adaptada de Stankiewicz, en nuestra serie contamos con un caso de hematoma orbitario y dos casos de hemorragias con compromiso hemodinámico que requirieron transfusión sanguínea, lo que implica una incidencia de $0,51 \%$, asimilable a los datos de centros especializados ${ }^{2}$.

Sobre el tipo de complicación más frecuente, si bien varía según la serie reportada, suelen encontrarse dentro de ellas las hemorrágicas y orbitarias, como en nuestra serie. A diferencia de estos reportes, no encontramos fístulas de líquido cerebroespinal en nuestro análisis. Es importante destacar que todos los pacientes son evaluados con tomografía computada previo a la cirugía, lo que permite una adecuada planificación quirúrgica y análisis anatómico, concordante con lo recomendado internacionalmente ${ }^{1}$.

\section{Con respecto a las complicaciones analizadas en esta serie:}

\section{a) Hemorrágicas}

Fueron la principal causa de cirugía incompleta. Con respecto al origen del sangrado, si bien se describe que este se puede explicar por daño arterial a nivel de arterias etmoidales, arteria esfenopalatina y arteria carótida inter$\mathrm{na}^{7,14-16}$ (siendo esta última la más grave alcanzando mortalidad de hasta $17 \%{ }^{17}$ ); los casos de esta serie se presentaron en contexto de patología inflamatoria, con sangrado difuso de la mucosa nasal por lo que el manejo fue variado, siendo utilizado taponamiento mecánico con merocel, agentes hemostáticos como surgiflo o surgicel y electrocauterio. En ningún caso se realizó ligadura de arteria etmoidal anterior o esfenopalatina. Solo dos casos de hemorragia severa requirieron transfusión de unidades de glóbulos rojos: el primero se trató de un nasofibroangioma previamente embolizado, y el segundo caso se dio en una cirugía prolongada (360 min) en contexto RSC primaria difusa con pólipos, en la que se realizó Draf III.

\section{b) Hematoma orbitario}

Este puede ser causado por sangrado venoso o arterial, aunque la causa más frecuente es daño en la arteria etmoidal anterior en el techo etmoidal ${ }^{18}$. El diagnóstico es clínico, se observa exoftalmo progresivo, quemosis, dolor, alteraciones en la oculomotilidad, reacción pupilar enlentecida, alteraciones en agudeza visual ${ }^{7}$. En el caso presentado en nuestra serie, al finalizar la cirugía se evidenció un aumento de volumen periocular asociado a quemosis. Entre 
las medidas generales iniciales descritas para su manejo se incluyen medidas básicas como posicionar la cabecera de la camilla en $45^{\circ}$, administración de hielo local y antibioterapia, no inducir valsalva, realizar masaje ocular en el ojo afectado, todo esto mientras se realiza interconsulta a oftalmología ${ }^{18}$. El manejo definitivo varía según si la agudeza visual se ve afectada o no: en caso de que no se vea alterada puede realizarse tratamiento con diuréticos endovenosos como la acetazolamida, aunque esto tiene un efecto más lento. Si la visión está afectada se tiene entre 60 a 90 minutos para realizar tratamiento, cuyo objetivo será drenar el hematoma; comúnmente se realiza descompresión de la órbita ya sea por vía endonasal, transoral o transconjuntival o con realización de orbitotomía a través de cantotomía y cantolisis ${ }^{7}$. Además, se debe manejar la hemorragia. En nuestro caso al ser detectado en pabellón, se retiraron de forma inmediata los tapones de ambas fosas nasales y se revisó la hemostasia hallando un vaso periorbitario con sangrado activo que fue cauterizado con pinza bipolar de forma exitosa. Además, se realizó cantotomía externa con la ayuda del equipo de oftalmología, ya que se contaba con su disponibilidad en menos de 60 minutos. El paciente evolucionó sin secuelas. Estamos conscientes que contar con oftalmólogo con disponibilidad inmediata no es la realidad en todos los centros de nuestro país, por lo que destacamos la importancia de realizar la cantotomía y cantolisis como otorrinolaringólogos de forma prioritaria ante un hematoma orbitario, ya que son estas las medidas de mayor impacto en la disminución de la presión intraocular y por ende en el pronóstico visual del paciente. En la literatura también se describen el uso de fármacos como manitol y corticoides, los cuales no siempre son efectivos ${ }^{19} \mathrm{y}$ no se utilizaron como tratamiento del hematoma en el caso descrito.

\section{c) Exposición de grasa periorbitaria hacia la cavidad nasal}

A diferencia de los trabajos internacionales, en el presente trabajo se incluyó dentro de las complicaciones aquellos casos en los que hubo dehiscencia de lámina papirácea con exposición de grasa periorbitaria hacia la cavidad nasal. Se decidió incluir esto como complicación debido a que encontramos que durante una CEN la lámina papirácea no debiese ser traspasada. Estos casos fueron detectados precozmente y confirmado con maniobras de presión ocular que evidenciaron el movimiento de la grasa. Todos los casos se manejaron de forma conservadora, teniendo especial precaución con respecto al uso de microdebridador en dicha zona.

Con respecto a las variables asociadas a complicaciones, el tiempo quirúrgico fue el único factor analizado que presentó significancia con respecto a esta asociación. Esto podría traducir qué cirugías más extensas tienen mayor riesgo de complicación o que, en los casos de hemorragia, esta misma sea causante de una cirugía más prolongada. Otra explicación sería que al producirse una complicación el cirujano opera más cautelosamente, lo cual enlentece la cirugía. También se podría considerar que un cirujano menos experimentado demora más tiempo, y por su curva de aprendizaje tiene más complicaciones. Con respecto a este último punto, al tratarse de un centro docente en el que en todas las cirugías participan residentes de distintos años de formación, es difícil precisar esta información en nuestra serie.

A diferencia de los reportes en la literatura, en los que las complicaciones se asocian más a la patología poliposa y lateralidad derecha, no encontramos asociación significativa en relación con el diagnóstico de base ni tampoco en la lateralidad. Podría explicarse por la baja tasa de complicaciones analizadas.

En todos los casos en los que se presentó alguna complicación, esta se detectó rápidamente y se manejó de forma precoz. Probablemente debido a esto es que ninguno de los pacientes de esta serie evolucionó con secuelas permanentes.

Con respecto al contexto nacional, si bien no existen trabajos que analicen complicaciones intraoperatorias de cirugía endoscópica nasosinusal propiamente tal, existen artículos en los que se analizan patologías puntuales tratadas por vía endoscópica. Arregui y cols. describen el tratamiento endoscópico de 180 pacientes con RSC primaria difusa con pólipos en los que no presentaron complicaciones mayores y su tasa de complicaciones menores fue de $9 \%$, con hemorragia como complicación más frecuente al igual que en nuestro trabajo, y si bien un paciente presentó fístula 
de líquido cerebroespinal no la consideraron una complicación mayor al ser reparada en el intraoperatorio ${ }^{20}$. Utilizando los criterios de nuestro trabajo esta habría correspondido a una complicación mayor, siendo la tasa de $0,55 \%$, muy similar a la obtenida en nuestro centro.

Santamaría y su equipo analizaron el manejo endoscópico de 57 pacientes con papiloma invertido, con una tasa global de complicaciones de 7,8\% correspondiente a cuatro pacientes ${ }^{21}$. De ellas dos $(3,5 \%)$ correspondieron a complicaciones intraoperatorias, ambas fístulas de líquido cerebroespinal, al tratarse de complicaciones mayores esta tasa se encuentra por sobre nuestra serie y la de Arregui, más los tamaños poblacionales no son comparables y existen otros factores no analizados que podrían aumentar dicha tasa, como por ejemplo la sincronía con carcinoma escamoso o si se trataba de reintervenciones.

Waissbluth y cols. describen el tratamiento endoscópico de 18 pacientes con papiloma invertido, en los que no presentaron complicaciones intraoperatorias ${ }^{22}$. En otro de sus trabajos analizan la realización de Draf III en 11 pacientes en los que tampoco hubo complicaciones intraoperatorias ${ }^{23}$. Por otra parte, Sáez y su equipo describen el manejo de 6 pacientes con nasofibroangioma, de los cuales 3 fueron operados por vía endoscópica sin presentar complicaciones ${ }^{24}$. Ya que la tasa de complicaciones intraoperatorias esperada es baja, su ausencia en dichas series se puede explicar por el pequeño número de pacientes analizados.

Las limitaciones de nuestro estudio es que corresponde a un estudio retrospectivo en que sólo analizamos los protocolos operatorios, dejando fuera la evolución de los pacientes, por lo cual no pudimos detectar complicaciones que se presentan en el posoperatorio como por ejemplo epífora o pérdida del olfato.

\section{Conclusión}

En estos últimos diez años se observa una tasa de complicaciones intraoperatorias en CEN baja en el Hospital del Salvador. Dentro de ellas, las más frecuentes son las hemorrágicas y las orbitarias. Finalmente, con este análisis establecemos el primer reporte a nivel nacional sobre las complicaciones intraoperatorias de la CEN.

\section{Bibliografía}

1. Lobo DR, López-Cortijo C, de la Fuente R, Laguna D, Pinilla M, Górriz C. Cirugía endoscópica nasosinusal: revisión de 1.093 casos [Endoscopic sinus surgery. A review of 1,093 cases]. Acta Otorrinolaringol Esp. 2003;54(6):435-440. doi: 10.1016/s00016519(03)78433-7.

2. Bross S, Schimelmitz J, Arrieta J, Guzmán R: Complicaciones y causas de falla en cirugía endoscópica nasosinusal. An Med Asoc Med Hosp ABC. 1999; 44 (2): 77-81.

3. Stankiewicz JA. Complications of endoscopic intranasal ethmoidectomy. Laryngoscope. 1987;97(11):1270-1273. doi: 10.1288/00005537198711000-00004.

4. Stankiewicz JA. Complications in endoscopic intranasal ethmoidectomy: an update. Laryngoscope. 1989;99:686-690. doi: 10.1288/00005537-19890700000004 .

5. Stankiewicz JA, Girgis S. Endoscopic surgical treatment of nasal and paranasal sinus inverted papilloma. Otolaryngol Head Neck Surg. 1993;109(6):988-995. doi: 10.1177/019459989310900603

6. Vargas-Aguayo AM, Copado-Ceballos RE, VivarAcevedo E, et al. Cirugía endoscópica de nariz y senos paranasales Complicaciones en 150 pacientes. Rev Med Inst Mex Seguro Soc. 2014;52(2):134-137.

7. Hosemann W, Draf C. Danger points, complications and medico-legal aspects in endoscopic sinus surgery. GMS Curr Top Otorhinolaryngol Head Neck Surg. 2013;12:Doc06. doi: 10.3205/cto000098.

8. May M, Levine HL, Mester SJ, Schaitkin B. Complications of endoscopic sinus surgery: analysis of 2108 patients--incidence and prevention. Laryngoscope. 1994;104(9):1080-1083. doi: 10.1288/00005537-199409000-00006

9. Dessi P, Castro F, Triglia JM, Zanaret M, Cannoni M. Major complications of sinus surgery: a review of 1192 procedures. J Laryngol Otol. 1994;108(3):212215. doi: $10.1017 / \mathrm{s} 0022215100126325$

10. Hopkins C, Browne JP, Slack R, et al. Complications of surgery for nasal polyposis and chronic rhinosinusitis: the results of a national audit in England and Wales. Laryngoscope.2006;116(8):1494-1499. doi: 10.1097/01.mlg.0000230399.24306.50.

11. Guerrero J, Molina B, Echeverría L, Arribas I, Rivera T. Cirugía endoscópica nasosinusal: estudio de 110 pacientes con rinosinusitis crónica con pólipos 
[Endoscopic sinonasal surgery: study of 110 patients with nasal polyposis and chronic rhinosinusitis]. Acta Otorrinolaringol Esp. 2007;58(6):252-256.

12. Maniglia AJ. Fatal and major complications secondary to nasal and sinus surgery. Laryngoscope. 1989;99(3):276-283. doi: 10.1288/00005537198903000-00008.

13. Gross RD, Sheridan MF, Burgess LP. Endoscopic sinus surgery complications in residency. Laryngoscope. 1997;107(8):1080-1085. doi: 10.1097/00005537-199708000-00014.

14. Moon HJ, Kim HU, Lee JG, Chung IH, Yoon JH. Surgical anatomy of the anterior ethmoidal canal in ethmoid roof. Laryngoscope. 2001;111(5):900-4. doi: 10.1097/00005537-200105000-00027.

15. Moeller CW, Welch KC. Prevention and management of complications in sphenoidotomy. Otolaryngol Clin North Am. 2010;43(4):839-54. doi: 10.1016/j. otc.2010.04.009.

16. Bouthillier A, van Loveren HR, Keller JT. Segments of the internal carotid artery: a new classification.

Neurosurgery. 1996;38(3):425-32;432-3. doi: 10.1097/00006123-199603000-00001.

17. Pepper JP, Wadhwa AK, Tsai F, Shibuya T, Wong BJ. Cavernous carotid injury during functional endoscopic sinus surgery: case presentations and guidelines for optimal management. Am J Rhinol. 2007;21:105-9. doi: 10.2500/ajr.2007.21.2901.

18. Moralee S. Should prophylactic antibiotics be used in the management of cerebrospinal fluid rhinorrhoea following endoscopic sinus surgery? A review of the literature. Clin Otolaryngol Allied Sci. 1995;20(2):100-
2. doi: 10.1111/j.1365-2273.1995.tb00023.x.

19. Stankiewicz JA, Lal D, Connor M, Welch K. Complications in endoscopic sinus surgery for chronic rhinosinusitis: a 25-year experience. Laryngoscope. 2011;121(12):2684-701. doi: 10.1002/ lary.21446.

20. Arregui R, Osorio J, Readi A, Barría T. Manejo quirúrgico de la poliposis nasosinusal. Rev Otorrinolaringol Cir Cabeza Cuello. 2010;70(1):7-16. doi: 10.4067/S0718-48162010000100002

21. Santamaria A, Tapia C, Fredes F, Astudillo D, Mendoza I. Manejo endoscópico de papiloma invertido nasosinusal. Experiencia de 15 años en Hospital Clínico Regional de Concepción. Rev Otorrinolaringol Cir Cabeza Cuello. 2016;76; 155-159. doi: 10.4067/S0718-48162016000200002.

22. Waissbluth S, García K, Imarai C. Cirugía endoscópica nasal en el tratamiento de papiloma invertido: A propósito de 18 casos. Rev Otorrinolaringol Cir Cabeza Cuello. 2018;78(2):127132. doi: 10.4067/s0717-75262018000200127.

23. Waissbluth S, Muñoz T, Maul X, González C, Callejas C. Abordaje endoscópico del seno frontal mediante Draf III. Experiencia de la Red de Salud UC Christus. Rev Otorrinolaringol Cir Cabeza Cuello. 2019;79(1):50-58. doi: 10.4067/s071848162019000100050 .

24. Sáez E, Medel S, Fernández F, Cox P. Angiofibroma nasofaríngeo juvenil: Estudio de 6 casos en el Hospital Carlos van Buren de Chile. Rev Otorrinolaringol Cir Cabeza Cuello. 2016;76(1):43-50. doi: 10.4067/s071848162016000100006. 